\title{
A REMARKABLE CLASS OF CONTINUED FRACTIONS ${ }^{1}$
}

\author{
WILLIAM W. ADAMS AND J. L. DAVISON
}

\begin{abstract}
For any irrational number $\alpha$ and integer $a>1$, the continued fraction of $(a-1) \sum_{r=1}^{\infty} 1 / a^{[r \alpha]}$ is computed explicitly in terms of the continued fraction of $\alpha$.
\end{abstract}

1. Introduction. In [2], the second author proved the remarkable result that $\sum_{r=1}^{\infty} 1 / 2^{[r \alpha]}$ where $\alpha=(1+\sqrt{5}) / 2$ has as its continued fraction $\left[0,1, t_{2}, t_{3}, \ldots\right]$ where $t_{n}=2^{f_{n-2}}(n \geqslant 2)$ and $f_{n}$ is the $n$th Fibonacci number. In this paper we generalize this result.

Let $\alpha>0$ be any real number and let $a>1$ be an integer. ${ }^{2}$ Define

$$
S_{a}(\alpha)=(a-1) \sum_{r=1}^{\infty} \frac{1}{a^{[r \alpha]}} \text {. }
$$

Consider the continued fraction for $\alpha^{-1}$,

$$
\alpha^{-1}=\left[a_{0} ; a_{1}, a_{2}, \ldots\right] \text {. }
$$

(We use the usual continued fraction notation, say for example in [3].) Let $p_{n} / q_{n}$ denote the convergents of $\alpha^{-1}$. We adopt the convention throughout this paper that $p_{-2}=0, p_{-1}=1, q_{-2}=1, q_{-1}=0$. Then for all $n \geqslant 0$,

$$
p_{n}=a_{n} p_{n-1}+p_{n-2} \text { and } q_{n}=a_{n} q_{n-1}+q_{n-2} \text {. }
$$

Define another sequence of integers

$$
t_{0}=a_{0} a, \quad t_{n}=\left(a^{q_{n}}-a^{q_{n-2}}\right) /\left(a^{q_{n-1}}-1\right) \text { for } n \geqslant 1 .
$$

( $t_{n}$ is an integer since $q_{n-1} \mid q_{n}-q_{n-2}$ )

THEOREM. If $\alpha$ is irrational and $\alpha>0$, then

$$
S_{a}(\alpha)=\left[t_{0} ; t_{1}, t_{2}, \ldots\right] \text {. }
$$

We will also give explicit formulas for convergents $P_{n} / Q_{n}$ of $S_{a}(\alpha)$. For this we need to define for real $x$,

$$
[x]^{\prime}= \begin{cases}{[x],} & \text { if } x \text { is not an integer, } \\ x-1, & \text { if } x \text { is an integer, }\end{cases}
$$

Received by the editors November 30, 1976.

AMS (MOS) subject classifications (1970). Primary 10A30, 10F20.

1 The results in this paper were obtained independently by the two authors. The second author would like to thank M. Mendes France for pointing out that the " 2 " in his first paper could be replaced by a variable " $a$ ".

${ }^{2}$ Any $a$ with $|a|>1$ yields the same result. 
the largest integer $<x$. Further define

$$
S_{a}^{\prime}(\alpha)=(a-1) \sum_{r=1}^{\infty} \frac{1}{a^{[r \alpha]}} .
$$

(Of course, for irrational $\alpha, S_{a}(\alpha)=S_{a}^{\prime}(\alpha)$.)

Proposition. If $\alpha$ is irrational, $p_{n} / q_{n}$ are the convergents of $\alpha^{-1}$ and $P_{n} / Q_{n}$ are the convergents of $S_{a}(\alpha)$, then for $n \geqslant 0$,

$$
\frac{P_{n}}{Q_{n}}= \begin{cases}S_{a}^{\prime}\left(q_{n} / p_{n}\right), & n \text { even, } \\ S_{a}\left(q_{n} / p_{n}\right), & n \text { odd }\end{cases}
$$

(if $p_{0}=0$ interpret $S_{a}^{\prime}\left(q_{0} / p_{0}\right)=0$ ).

Also

$$
P_{n}= \begin{cases}\sum_{t=1}^{p_{n}} a^{q_{n}-\left[t q_{n} / p_{n}\right]}, & n \text { even, } \\ \sum_{t=1}^{p_{n}} a^{q_{n}-\left[t q_{n} / p_{n}\right],} & n \text { odd },\end{cases}
$$

and

$$
Q_{n}=\left(a^{q_{n}}-1\right) /(a-1) .
$$

In the last section we observe that $S_{a}(\alpha)$ is transcendental.

2. Lemmas. We assume throughout that $\alpha$ is irrational.

Lemma 1. Assume $1 \leqslant r<p_{n+1}$ and $n \geqslant 0$. Then

$$
[r \alpha]= \begin{cases}{\left[r q_{n} / p_{n}\right]^{\prime},} & n \text { even, } \\ {\left[r q_{n} / p_{n}\right],} & n \text { odd }\end{cases}
$$

( for $n=0$ assume $\left.p_{0} \neq 0\right)$.

Proof. The $n$th convergent to $\alpha^{-1}$ is $p_{n} / q_{n}$ and thus $q_{n} / p_{n}$ is the $n+1$ or $n-1$ (depending on whether $a_{0}=0$ or $a_{0} \neq 0$, respectively) convergent of $\alpha$. Thus

$$
\alpha=q_{n} / p_{n}+\theta_{n} / p_{n} p_{n+1},
$$

where $0<\theta_{n}<1$ if $n$ is odd and $-1<\theta_{n}<0$ if $n$ is even. Hence, $1<r<$ $p_{n+1}$ implies

$$
r \alpha=r q_{n} / p_{n}+\theta_{n}^{\prime} / p_{n},
$$

where $0<\theta_{n}^{\prime}<1$ if $n$ is odd and $-1<\theta_{n}^{\prime}<0$ if $n$ is even. If $p_{n} \nmid r$, then there is no integer between $r q_{n} / p_{n}$ and $r \alpha$ and

If $p_{n} \mid r$, then

$$
[r \alpha]=\left[r q_{n} / p_{n}\right]=\left[r q_{n} / p_{n}\right]^{\prime} .
$$

$$
[r \alpha]=r q_{n} / p_{n}+\left[\theta_{n}^{\prime} / p_{n}\right]
$$

which immediately gives the desired result. 
Lemma 2. Assume $1 \leqslant r<p_{n}$ and $n \geqslant 0$. Then

$$
\begin{array}{ll}
{\left[r q_{n} / p_{n}\right]=\left[r q_{n-1} / p_{n-1}\right]^{\prime},} & n \text { odd, } \\
{\left[r q_{n} / p_{n}\right]^{\prime}=\left[r q_{n-1} / p_{n-1}\right],} & n \text { even. }
\end{array}
$$

Proof. Immediate from Lemma 1.

LEMMA 3.

$$
\lim _{\substack{n \rightarrow \infty \\ n \text { odd }}} S_{a}\left(\frac{q_{n}}{p_{n}}\right)=\lim _{\substack{n \rightarrow \infty \\ n \text { even }}} S_{a}^{\prime}\left(\frac{q_{n}}{p_{n}}\right)=S_{a}(\alpha) .
$$

Proof. If $n$ is even, we have by Lemma 1,

$$
\left|S_{a}^{\prime}\left(\frac{q_{n}}{p_{n}}\right)-S_{a}(\alpha)\right|=(a-1)\left|\sum_{r=p_{n+1}}^{\infty}\left(a^{-\left[r q_{n} / p_{n}\right]^{\prime}}-a^{-[r \alpha]}\right)\right|
$$

and it is trivial to see that this tends to zero. The situation is similar if $n$ is odd.

LEMMA 4.

$$
\begin{aligned}
& S_{a}\left(\frac{q_{n}}{p_{n}}\right)=\frac{a-1}{a^{q_{n}}-1} \sum_{t=1}^{p_{n}} a^{q_{n}-\left[t q_{n} / p_{n}\right]} . \\
& S_{a}^{\prime}\left(\frac{q_{n}}{p_{n}}\right)=\frac{a-1}{a^{q_{n}}-1} \sum_{t=1}^{p_{n}} a^{q_{n}-\left[t q_{n} / p_{n}\right]^{\prime}} .
\end{aligned}
$$

Proof. In definition (3) of $S_{a}^{\prime}\left(q_{n} / p_{n}\right)$, set $r=k p_{n}+t, 1 \leqslant t \leqslant p_{n}, 0 \leqslant k$ $<\infty$. Thus

$$
\begin{aligned}
S_{a}^{\prime}\left(\frac{q_{n}}{p_{n}}\right) & =(a-1) \sum_{k=0}^{\infty} \sum_{t=1}^{p_{n}} a^{-\left[k q_{n}+t q_{n} / p_{n}\right]} \\
& =(a-1) \sum_{t=1}^{p_{n}} a^{-\left[t q_{n} / p_{n}\right]} \sum_{k=0}^{\infty} a^{-k q_{n}} \\
& =(a-1)\left(\sum_{t=1}^{p_{n}} a^{-\left[t q_{n} / p_{n}\right]}\right)\left(1-\frac{1}{a^{q_{n}}}\right)^{-1}
\end{aligned}
$$

which is the desired result. $S_{a}\left(q_{n} / p_{n}\right)$ is similar.

3. Proof of the theorem ${ }^{3}$ and proposition. From Lemmas 3 and 4 we see that it suffices to show that the quantities on the right-hand sides of (4) and (5) satisfy the appropriate recursion relations. Let $P_{n}^{\prime}$ denote the right side of (4) and $Q_{n}^{\prime}$ denote the right side of $(5)(n \geqslant 0)$.

Set $Q_{-2}^{\prime}=1, Q_{-1}^{\prime}=0$. We need that for all $n \geqslant 0$,

$$
Q_{n}^{\prime}=t_{n} Q_{n-1}^{\prime}+Q_{n-2}^{\prime}
$$

where $t_{n}$ is given in (2). For $n=0$ this is clear. For $n=1$,

${ }^{3}$ This theorem was already proved by Loxton and Van der Poorten (see [4], [5]). Their proof resembles, in much less explicit form, our proof for the continued fraction identity. 


$$
t_{1} Q_{0}^{\prime}+Q_{-1}^{\prime}=t_{1}=\left(a^{q_{1}}-a^{q_{-1}}\right) /\left(a^{q_{0}}-1\right)=Q_{1}^{\prime} .
$$

For $n \geqslant 2$, we have by induction

$$
\begin{aligned}
t_{n} Q_{n-1}^{\prime}+Q_{n-2}^{\prime} & =\frac{a^{q_{n}}-a^{q_{n-2}}}{a^{q_{n-1}}-1} \frac{a^{q_{n-1}}-1}{a-1}+\frac{a^{q_{n-2}}-1}{a-1} \\
& =\left(a^{q_{n}}-1\right) /(a-1)=Q_{n}^{\prime}
\end{aligned}
$$

as desired.

Set $P_{-2}^{\prime}=0, P_{-1}^{\prime}=1$. We need that for all $n \geqslant 0$,

$$
P_{n}^{\prime}=t_{n} P_{n-1}^{\prime}+P_{n-2}^{\prime} \text {. }
$$

If $n=0$, then $t_{n} P_{n-1}^{\prime}+P_{n-2}^{\prime}=t_{0}=a_{0} a$, whereas

$$
P_{0}^{\prime}=\sum_{t=1}^{p_{0}} a^{q_{0}-\left[t q_{0} / p_{0}\right]^{\prime}}=\sum_{t=1}^{a_{0}} a^{1-\left[t / a_{0}\right]^{\prime}}=a_{0} a .
$$

(If $a_{0}=0$, this is still valid.)

If $n=1$,

$$
t_{1} P_{0}^{\prime}+P_{-1}^{\prime}=a_{0} a \frac{a^{q_{1}}-a^{q_{-1}}}{a^{q_{0}}-1}+1=a_{0} a \frac{a^{a_{1}}-1}{a-1}+1 .
$$

Also

$$
\begin{aligned}
P_{1}^{\prime} & =\sum_{t=1}^{p_{1}} a^{q_{1}-\left[t q_{1} / p_{1}\right]}=\sum_{t=1}^{p_{1}-1} a^{q_{1}-\left[t q_{0} / p_{0}\right]^{\prime}}+1 \\
& =\sum_{t=1}^{p_{1}-1} a^{a_{1}-\left[t / a_{0}\right]^{\prime}}+1,
\end{aligned}
$$

using Lemma 2. (If $a_{0}=0$ the result is clear.) It is easily seen that the numbers $\left[t / a_{0}\right]^{\prime}, 1 \leqslant t \leqslant p_{1}-1=a_{1} a_{0}$, give $a_{0}$ repetitions of each of the numbers $0,1,2, \ldots, a_{1}-1$. Hence

$$
P_{1}^{\prime}=a_{0} \sum_{j=0}^{a_{1}-1} a^{a_{1}-j}+1
$$

which is the same as (7).

Now suppose $n>1$. We verify (6) in the case that $n$ is even; the case where $n$ is odd is similar. From Lemma 2 and (4)

$$
P_{n}^{\prime}=\sum_{t=1}^{p_{n}-1} a^{q_{n}-\left[t q_{n-1} / p_{n-1}\right]}+a
$$

Set $t=i p_{n-1}+j, 1 \leqslant j \leqslant p_{n-1}$. Then

$$
1 \leqslant t \leqslant p_{n}-1=a_{n} p_{n-1}+p_{n-2}-1
$$

if and only if

$$
0 \leqslant i \leqslant a_{n}-1 \text { and } 1 \leqslant j \leqslant p_{n-1}
$$

or

$$
i=a_{n} \quad \text { and } \quad 1 \leqslant j \leqslant p_{n-2}-1 .
$$

Thus 


$$
\begin{aligned}
& P_{n}^{\prime}=\sum_{i j} a^{q_{n}-i q_{n-1}-\left[j q_{n-1} / p_{n-1}\right]}+a \\
& =\sum_{j=1}^{p_{n-1}} a^{q_{n}-\left[j q_{n-1} / p_{n-1}\right]} \sum_{i=0}^{a_{n}-1} a^{-i q_{n-1}} \\
& +\sum_{j=1}^{p_{n-2}-1} a^{q_{n}-a_{n} q_{n-1}-\left[j q_{n-1} / p_{n-1}\right]}+a \\
& =\frac{a^{-a_{n} q_{n-1}}-1}{a^{-q_{n-1}}-1} \sum_{j=1}^{p_{n-1}} a^{q_{n}-\left[j q_{n-1} / p_{n-1} \mathrm{]}\right.}+\sum_{j=1}^{p_{n-2}-1} a^{q_{n-2}-\left[j q_{n-1} / p_{n-1}\right]}+a \\
& =\frac{a^{q_{n}}-a^{q_{n-2}}}{a^{q_{n-1}}-1} \sum_{j=1}^{p_{n-1}} a^{q_{n-1}-\left[j q_{n-1} / p_{n-1}\right]} \\
& +\sum_{j=1}^{p_{n-2}-1} a^{q_{n-2}-\left[j q_{n-2} / p_{n-2}\right]^{\prime}}+a \quad \text { (by Lemma 2) } \\
& =t_{n} P_{n-1}^{\prime}+P_{n-2}^{\prime}
\end{aligned}
$$

by induction. The proof is complete.

4. The transcendence of $S_{a}(\alpha)$. From Roth's theorem [1, p. 104] we have that if $|q \beta-p|<1 / q^{1.4}$ has an infinite number of integer solutions $q, p$, then $\beta$ is transcendental. If $A_{n} / B_{n}$ are the convergents of $\beta$ then

$$
\left|B_{n} \beta-A_{n}\right|<1 / B_{n+1} \leqslant 1 / B_{n}^{1.4}
$$

provided $\log B_{n+1} / \log B_{n} \geqslant 1.4$. For $B_{n}=Q_{n}$ above we have

$$
\log Q_{n+1} / \log Q_{n} \sim q_{n+1} / q_{n} \quad(n \rightarrow \infty) .
$$

It is easily checked that $\max \left(q_{n+1} / q_{n}, q_{n} / q_{n-1}\right) \geqslant 1.5$. Thus

THEOREM. $S_{a}(\alpha)$ is transcendental for all irrationals $\alpha$.

\section{REFERENCES}

1. J. W. S. Cassels, An introduction to diophantine approximations, Cambridge Univ. Press, Cambridge, 1957.

2. J. L. Davison, A series and its associated continued fraction, Proc. Amer. Math. Soc. 63 (1977), 29-32.

3. S. Lang, Introduction to diophantine approximations, Addison-Wesley, Reading, Mass., 1966.

4. J. H. Loxton and A. J. Van der Poorten, Arithmetric properties of certain functions of several variables. III, Bull. Austral. Math. Soc. 16 (1977), 15-49.

5. $\longrightarrow$ Transcendence theory: Advances and applications, Academic Press, New York, 1977, pp. 211-226.

Department of Mathematics, University of Maryland, College Park, Maryland 20742

Department of Mathematics, Laurentian University, Sudbury, Ontario, Canada 